\title{
Living well with kidney disease
}

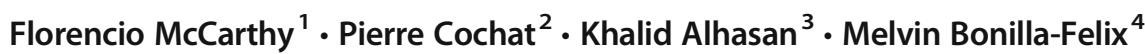

Received: 15 February 2021 / Accepted: 15 February 2021 / Published online: 1 March 2021

(C) IPNA 2021

World kidney day is a global kidney health awareness campaign that happens yearly every second Thursday in March. As in the last 16 years, the International Pediatric Nephrology Association (IPNA) joins in the celebration of World Kidney Day on March 11, 2021.

The objectives of this celebration include:

1. Increase awareness of how important our kidneys are

2. Highlight that diabetes and high blood pressure are key risk factors for chronic kidney disease

3. Encourage systematic kidney evaluation of all patients with diabetes and hypertension

4. Promote preventive behaviors

5. Awareness of how to live well with kidney disease

6. Promote the importance of preventing and treating childhood kidney disease

7. Educate healthcare professionals about their key roles in detecting and reducing the risk of kidney disease, especially in high-risk populations

8. Encourage transplantation as the best outcome option for chronic kidney disease and promote organ donation

Chronic kidney disease (CKD) is a non-communicable disease that affects 1 in 10 people in the world, including the pediatric population. While the severity can vary, CKD is incurable and requires the patient to receive lifelong medical

Florencio McCarthy

famw1997@gmail.com

1 Hospital del Niño Dr. José Renán Esquivel, Calle 34 Este, Panamá City, Panama

2 Centre de référence des maladies rénales rares Néphrogones, Hospices Civils de Lyon \& Université Claude-Bernard Lyon 1, Lyon, France

3 Pediatrics Department, College of Medicine, King Saud University, Riyadh, Kingdom of Saudi Arabia

4 Department of Pediatrics, University of Puerto Rico, Medical Sciences Campus, PO Box 365067, San Juan 00936-5067, Puerto Rico care. Due to the increase in the incidence of kidney disease, the celebration of World Kidney Day plays an important role in educating the public, medical community, and policymakers in encouraging the prevention and early detection of kidney disease.

Chronic kidney disease is rare in children but has a major impact on growth, hemoglobin levels, blood pressure, cardiovascular health, mineral and bone metabolism, and mental health. Although accurate epidemiological data are difficult to come by, especially in low-resource countries, and also because most of the data are obtained from dialysis centers, leading to underreporting of pre-dialysis cases, the incidence per million age-related population including early stages of CKD is estimated to be 11-12 per million in Europe, 2.815.8 per million in Latin America, 38 per million in Asia, and 1-3 per million in Africa. The prevalence ranges from 55 to 75,329 , and 20 per million age-related populations in Europe, Asia, and Brazil, respectively [1, 2].

To improve the quality of life of our patients, it is critical to increase awareness of the importance of kidney diseases. For instance, despite the major impact that kidney disease has on cardiovascular health, the World Health Organization Global Action Plan for the Prevention and Control of Non-communicable Diseases 2013-2020 does not mention CKD. This underscores the need for continuous education and lobbying of policy makers. Children with kidney disease, especially those living in limited-resource regions, are an extremely vulnerable population. As the prevalence of CKD in children is much lower than in adults, they often remain invisible to those in charge of allocating resources. Many countries that have established kidney replacement programs for adults do not support pediatric programs. Although kidney transplantation is recognized as the optimal therapy for stage $5 \mathrm{CKD}$, it is not available in many low-income countries, and when available, it is limited to organs from living-related donors.

Chronic kidney disease may have an impact on cognitive development of children and their quality of life [3]. Patients have a lower level of intellectual functioning and memory, 
attention deficits, and executive functioning deficits [4]. In addition, the physical effects of the disease, mainly on growth, can have an effect on the self-esteem of these patients. Kidney disease also affects the social life of those who suffer from it by limiting their school activities, the practice of sports, or social events with children of their age [5]. Although children and adolescents with CKD may have levels of resilience similar to their peers, they show higher levels of separation anxiety and a higher frequency of significant clinical symptoms of depression [6].

This year, the IPNA World Kidney Day Steering Committee establishes the theme for the celebration: "Living Well With Kidney Disease" with the intention of increasing patient education, awareness, and evolving from a diseasecentered model to a patient-centered approach. This patientcentered model seeks to include the patient and their caregiver in the decisions that involve the care of the children with kidney disease, mainly allowing them to have a greater influence on those aspects that could interfere in the development of their daily activities, in such a way that they be allowed to have greater control of their environment and their daily activities while maintaining adequate care for their health but also for their quality of life.

Last year we received eighteen nominations to the IPNA World Kidney Day campaign, all of very high quality and from different regions of the world. In a very close selection, two projects were the winners for the first time in the history of this campaign:

- Prospective study on incidence and risk factors for Severe Pediatric Acute Kidney Injury by Dr. Azmeri Sultana (Dhaka, Bangladesh)

- Educating community for prevention of kidney diseases project by Dr. Girish Bhatt (Bhopal, India)
Both received financial support from IPNA for their development.

The IPNA Council encourages our members to actively participate in the celebration of this World Kidney Day by carrying out, as in previous years, activities that promote better access to care and meet the objectives established for this year of empowering our young patients and their caregivers to achieve their life goals, promoting a comprehensive treatment that, in addition to the traditional treatments for kidney disease, includes treatment of the neurocognitive, social, and psychological consequences of the disease so that they can have a better quality of life.

\section{References}

1. Harambat J, van Stralen KJ, Kim JJ, Tizard EJ (2012) Epidemiology of chronic kidney disease in children. Pediatr Nephrol 27:363-373

2. Konstantyner T, Sesso R, de Camargo MF, de SantisFeltran L, Koch-Nogueira PC (2015) Pediatric chronic dialysis in Brazil: epidemiology and regional inequalities. PLoS One 10:e135649. https://doi.org/10.1371/journal.pone.0135649

3. Kaspar CDW, Bholah R, Bunchman TE (2016) A review of pediatric chronic kidney disease. Blood Purif 41:211-217

4. Chua A, Warady B (2017) Care of the pediatric patient on chronic dialysis. Adv Chronic Kidney Dis 24:388-397

5. Assadi F (2013) Psychological impact of chronic kidney disease among children and adolescents: not rare and not benign. J Nephropathol 2:1-3

6. Moreira JM, Bouissou Morais Soares CM, Teixeira AL, Simões E, Silva AC, Kummer AM (2015) Anxiety, depression, resilience and quality of life in children and adolescents with pre-dialysis chronic kidney disease. Pediatr Nephrol 30:2153-2162. https://doi.org/10. 1007/s00467-015-3159-6

Publisher's note Springer Nature remains neutral with regard to jurisdictional claims in published maps and institutional affiliations. 